\title{
主手征模型中隐藏对称代数中的 多重对易关系
}

葛墨林 王均义

(兰州大学物理系)
吴詠时

（中国科学院理论物理研究所, 北京)

近年来人们对两维可积系统的研究比较感兴趣, 特别是对两维主手征模型的讨论更是深 $\lambda^{[1]}$, 这不但是因为它具有非线性微分方程的一般特点, 而且由于它可能是通向研究杨-Mills 场理论的新途径的必经之路. 在长期研究的基础上 ${ }^{[2-4]}$, 现在对于与主手模型相关的问题 又 有了不少新的理解,这些理解导致了 $G \otimes C(t)$ 代数结构的出现 ${ }^{[5-8)}$.

对于代数结构, 尚有两点须要进一步考虑, 一个是向 $G \otimes C\left(t, t^{-1}\right)$ 代数的推广, 另一方面 是计算 $G \otimes C(t)$ 代数的多重对易子. 本文着重讨论后者.

主手征场的拉氏函数密度为

$$
\mathscr{L}=\operatorname{Tr}\left(\partial_{\mu} g \partial^{\mu} g^{-1}\right), \quad(\mu=0,1)
$$

在 $\mathrm{g}$ 作 $\mathrm{H}$-变换时, 即 ${ }^{[3}$

$$
\delta g=-g \Lambda(x)--g U(x) T U^{-1}(x),
$$

其中 $T-T \alpha^{*}, T a$ 为群 $G$ 生成元. 当 $U$ 满足线性方程

$$
\begin{aligned}
& \partial_{\xi} U=-\frac{l}{1+l} A_{\xi} U, A_{\xi}=g^{-1} \partial_{\xi} g \\
& \partial_{\eta} U=-\frac{l}{1-l}-A_{\eta} U, A_{\eta}=g^{-1} \partial_{\eta} g
\end{aligned}
$$

时 ( $\xi$ 与 $\eta$ 为光锥坐标), 则势 $A_{\xi}$ 与 $A_{\eta}$ 的变分满足与 $\delta g$ 类似的对易关系 ${ }^{01}$. 我们现在将这种 对易关系推广到多重对易子.

为了推广的方便，我们用更简单的方法推导势变分的单重对易子.

由文献 [9]知在主手征模型中, $H$-变换下存在一些关系,它们是(以 $\xi$ 指标为例)

$$
\begin{gathered}
\delta_{\alpha} A_{\xi}=\frac{1}{l} \partial_{\xi} \Lambda_{\alpha}, \delta_{\beta}^{\prime} A_{\xi}-\frac{1}{l^{\prime}} \partial_{\xi} \Lambda_{\alpha}^{\prime}, \\
\delta_{\beta}^{\prime} U U^{-1}=\frac{l}{l^{\prime}-l}\left(\Lambda_{\beta}-\Lambda_{\beta}^{\prime}\right), \delta_{\alpha} U^{\prime} U^{\prime-1}-\frac{l^{\prime}}{l^{\prime}-l}\left(\Lambda_{\alpha}-\Lambda_{\alpha}^{\prime}\right),
\end{gathered}
$$

其中记号同文献 [9]中的记号.

利用(4)式中的诸式可证

与

$$
\delta_{\alpha} \delta_{\beta}^{\prime} A_{\xi}-\frac{1}{l^{\prime}-l} \partial_{\xi}\left[\Lambda_{\alpha}-\Lambda_{a}^{\prime}, \Lambda_{\beta}^{\prime}\right]
$$

本文 1982 年9 月 24 日收到. 


$$
\delta_{p}^{\prime} \delta_{a} A_{\xi}-\frac{1}{l^{\prime}-l} \partial_{\xi}\left[\Lambda_{q}-\Lambda_{p}^{\prime}, \Lambda_{a}\right]
$$

遂得

$$
\left[\delta_{a}, \delta_{b}^{\prime}\right] \Lambda_{\xi}-\frac{1}{l-l^{\prime}}\left\{\left[\Lambda_{a}^{\prime}, \Lambda_{b}^{\prime}\right]-\left[\Lambda_{a}, \Lambda_{B}\right]\right\} .
$$

由(7)式推得

$$
\left[\delta_{a}, \delta_{\beta}^{\prime}\right] A_{\xi}-\alpha^{a} \beta^{b} C_{a b}^{c}\left\{\frac{l^{\prime}}{l-l^{\prime}} \delta_{c}^{\prime} A_{\xi}+\frac{l}{l^{\prime}-l} \delta_{c} A_{\xi}\right\} .
$$

它相应于

$$
\left[\delta_{a}^{(m)}, \delta_{b}^{(n)}\right] A_{\varepsilon}=-C_{a b}^{c} \delta_{c}^{(m+n)} A_{\xi},
$$

注意上面的推导中利用了

$$
\begin{aligned}
& \delta_{a}-\alpha^{a} \sum_{m} l^{m} \delta_{a}^{(n)}, \\
& \delta_{\beta}^{\prime}=\beta^{b} \sum_{n} l^{\prime n} \delta_{b}^{(n)},
\end{aligned}
$$

以及

$$
\sum_{m, n} l^{m} l^{\prime n} \cdots-\sum_{k=0}^{\infty} \sum_{m=0}^{k} l^{m} l^{k-m} \cdots .
$$

(12)式表示以 $m, n$ 为行列指标(包括 0,0 指标)的矩阵各元素求和.

以下我们计算三重变分式

$$
\left[\left[\delta_{a}, \delta_{\beta}^{\prime}\right], \delta_{r}^{\prime \prime}\right] A_{\xi}=\left[\delta_{\alpha}, \delta_{\beta}^{\prime}\right]\left(\delta_{r}^{\prime \prime} A_{\xi}\right)-\delta_{r}^{\prime \prime}\left(\left[\delta_{\alpha}, \delta_{\beta}^{\prime}\right] A_{\xi}\right) .
$$

应用前节结果可以得到

$$
\begin{aligned}
\delta_{r}^{\prime \prime}\left[\delta_{a}, \delta_{\beta}^{\prime}\right] A_{\xi}- & \alpha^{a} \beta^{b} C_{a b}^{c} \frac{1}{l-l^{\prime}}\left\{\frac{l^{\prime}}{l^{\prime}}-l^{\prime \prime} \partial_{\xi}\left[\Lambda_{r}^{\prime \prime}, \Lambda_{c}^{\prime}\right]\right. \\
& \left.+\frac{l}{l^{\prime \prime}-l} \partial_{\xi}\left[\Lambda_{r}^{\prime \prime}, \Lambda_{c}\right]\right\} \\
& +\alpha^{a} \beta^{b} \gamma^{d} C_{a b}^{c} C_{a c}^{\prime} \frac{1}{l^{\prime}-l}\left(\frac{l}{l^{\prime \prime}-l} \partial_{\xi} \Lambda_{f}+\frac{l^{\prime}}{l^{\prime}-l^{\prime \prime}} \partial_{\xi} \Lambda_{f}^{\prime}\right) .
\end{aligned}
$$

再利用(4)式可计算出

$$
\begin{aligned}
& {\left[\delta_{\alpha}, \delta_{\beta}^{\prime}\right] \Lambda_{\gamma}^{\prime \prime}-\frac{l^{\prime 2}}{\left(l-l^{\prime \prime}\right)\left(l^{\prime}-l^{\prime \prime}\right)} \alpha^{\alpha} \beta^{b} \gamma^{c} C_{a b}^{a} C_{\alpha c}^{e} \Lambda_{l}^{\prime \prime}} \\
& +l^{\prime \prime}\left[\frac{l^{\prime}}{\left(l-l^{\prime}\right)\left(l^{\prime}-i^{\prime \prime}\right)}-\frac{l}{\left(l-l^{\prime}\right)\left(l-l^{\prime \prime}\right)}\right]\left[\left[\Lambda_{\alpha}, \Lambda_{\beta}^{\prime}\right], \Lambda_{r}^{\prime \prime}\right] \\
& +\frac{l^{\prime \prime} l^{\prime \prime}}{\left(l-l^{\prime}\right)\left(l^{\prime \prime}-l^{\prime}\right)} \alpha^{a} \beta^{b} C_{\alpha u}^{d}\left[\Lambda_{d}^{\prime}, \Lambda_{r}^{\prime \prime}\right] \\
& +\frac{l l^{\prime \prime}}{\left(l-l^{\prime}\right)\left(l-l^{\prime \prime}\right)} a^{a} \beta^{b} C_{a c}^{d}\left[\Lambda_{d}, \Lambda_{\gamma}^{\prime \prime}\right] \\
& +\frac{l^{\prime \prime 2}}{\left(l-l^{\prime \prime}\right)\left(l^{\prime}-l^{\prime \prime}\right)}\left[\left[\Lambda_{\beta}^{\prime}, \Lambda_{r}^{\prime \prime}\right], \Lambda_{\alpha}\right] \\
& +\frac{l^{\prime \prime}}{\left(l-l^{\prime \prime}\right)\left(l^{\prime}-l^{\prime \prime}\right)}\left[\Lambda_{\beta}^{\prime},\left[\Lambda_{\alpha}, \Lambda_{r}^{\prime \prime}\right]\right] \text {. }
\end{aligned}
$$

利用雅可比恒等式,遂有 
$\left[\left[\delta_{a}, \delta_{\beta}^{\prime}\right], \delta_{r}^{\prime \prime}\right] A_{\xi}-\alpha^{e} \beta^{b} \gamma^{c} C_{e b}^{e} C_{e c}^{z}$

$$
\begin{aligned}
& \left\{\frac{l^{2}}{\left(l^{\prime}-l\right)\left(l^{\prime \prime}-l\right)} \delta_{g} A_{\xi}+\frac{l^{\prime 2}}{\left(l-l^{\prime}\right)\left(l^{\prime \prime}-l^{\prime}\right)} \delta_{\xi}^{\prime} A_{\xi}\right. \\
& \left.\quad+\frac{l^{\prime \prime 2}}{\left(l^{\prime}-l^{\prime \prime}\right)\left(l-l^{\prime \prime}\right)} \delta_{g}^{\prime \prime} A_{\xi}\right\} .
\end{aligned}
$$

将 $\delta_{g}, \delta_{g}^{\prime}$ 与 $\delta_{g}^{\prime \prime}$ 分别用参数 $l, l^{\prime}$ 与 $l^{\prime \prime}$ 展开有

$$
\begin{aligned}
& {\left[\left[\delta_{a}, \delta_{\beta}^{\prime}\right], \delta_{\gamma}^{\prime \prime}\right] A_{\xi}--\alpha^{a} \beta^{b} \gamma^{c} C_{a b}^{d} C_{a c}^{\alpha} \cdot} \\
& \quad \sum_{k}\left\{\frac{l^{\prime \prime k+2}-l^{k+2}}{\left(l-l^{\prime \prime}\right)\left(l^{\prime}-l^{\prime \prime}\right)}+\frac{l^{\prime k+2}-l^{k+2}}{\left(l-l^{\prime}\right)\left(l^{\prime \prime}-l^{\prime}\right)}\right\} \delta_{g}^{(k)} A_{\xi .}
\end{aligned}
$$

于是得到

$$
\left[\left[\delta_{\alpha}, \delta_{\beta}^{\prime}\right], \delta_{\gamma}^{\prime \prime}\right] A_{\xi}=\alpha^{a} \beta^{b} \gamma^{c} C_{a b}^{d} C_{d c}^{g} \sum_{k} \sum_{m=0}^{k+1} l^{m} \frac{l^{\prime \prime k-m+1}-l^{k-m+1}}{l^{\prime \prime}-l} \delta_{z}^{(k)} A_{\xi},
$$

因为当 $m=k+1$ 时贡献为零,故可将对 $m$ 求和号改为求和到 $k$, 我们有

$$
\begin{aligned}
& {\left[\left[\delta_{\alpha_{1}}, \delta_{\beta}^{\prime}\right], \delta_{r}^{\prime \prime}\right] A_{\xi}=\alpha^{a} \beta^{b} \gamma^{c} C_{\infty}^{d} C_{d c}^{g} .} \\
& \sum_{k} \sum_{m=0}^{k} l^{m} \sum_{n=0}^{k-m} l^{\prime n} l^{\prime \prime(k-m-n)} \delta g^{(k)} A_{\xi} .
\end{aligned}
$$

上式右端求和是立体形式的方阵沿斜截面等幂次对各元素求和,它等于简单的对各元素求和, 显然是二维方阵情况的推广. 于是我们得

同理,经过较繁的计算可得

$$
\left[\left[\delta_{a}^{(m)}, \delta_{b}^{(n)}\right], \delta_{c}^{(k)}\right] A_{\xi}-C_{a b}^{d} C_{d c}^{g} \delta_{z}^{(m+n+k)} A_{\xi} .
$$

$$
\begin{aligned}
& {\left[\left[\left[\delta_{a_{1}}\left(l_{1}\right), \delta_{a_{2}}\left(l_{2}\right)\right], \delta_{a_{3}}\left(l_{3}\right)\right], \delta_{a_{4}}\left(l_{4}\right)\right] A_{\xi}-\alpha_{1}^{a_{1} a_{2}^{a_{2}} \alpha_{3}^{a_{3}} \alpha_{4}^{a_{4}}} \cdot} \\
& \quad C_{a_{1} a_{2}}^{b_{1}} C_{b_{1} a_{3}}^{b_{2}} C_{b_{2} a_{4}}^{g}\left\{\frac{l_{1}^{3}}{\left(l_{2}-l_{1}\right)\left(l_{3}-l_{1}\right)\left(l_{4}-l_{1}\right)} \delta_{g}\left(l_{1}\right) A_{\xi}\right. \\
& \quad+\frac{l_{2}^{3}}{\left(l_{1}-l_{2}\right)\left(l_{3}-l_{2}\right)\left(l_{4}-l_{2}\right)} \delta_{g}\left(l_{2}\right) A_{\xi} \\
& \quad+\frac{l_{3}^{3}}{\left(l_{1}-l_{3}\right)\left(l_{2}-l_{3}\right)\left(l_{4}-l_{3}\right)} \delta_{g}\left(l_{3}\right) A_{\xi} \\
& \left.\quad+\frac{l_{4}^{3}}{\left(l_{1}-l_{4}\right)\left(l_{2}-l_{4}\right)\left(l_{3}-l_{4}\right)} \delta_{g}\left(l_{4}\right) A_{\xi}\right\},
\end{aligned}
$$

上式中为简单, 分别将任意参数记为 $l_{i}$, 并用到了记号

$$
\delta_{\alpha_{i}}\left(l_{i}\right)-\alpha_{i}^{a_{i}} \sum_{m_{i}} l^{m_{i}} \delta_{a_{i}}^{\left(m_{i}\right)} \text {. }
$$

经过计算, (21)式可化为

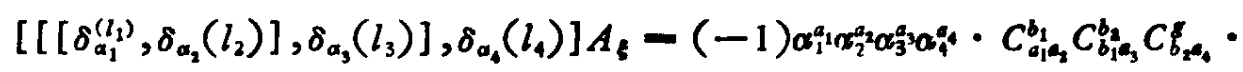

$$
\begin{aligned}
& \sum_{k} \sum_{m_{1}=0}^{k+2} l_{1}^{m_{1}}\left\{\frac{l_{2}^{(k+2)-m_{1}}}{\left(l_{3}-l_{2}\right)\left(l_{4}-l_{2}\right)}+\frac{l_{3}^{(k+2)-m_{1}}}{\left(l_{2}-l_{3}\right)\left(l_{4}-l_{3}\right)}\right. \\
& \left.+\frac{l_{4}^{(k+2)-m_{1}}}{\left(l_{2}-l_{4}\right)\left(l_{3}-l_{4}\right)}\right\} \cdot \delta_{8}^{(k)} A_{6} \text {. }
\end{aligned}
$$

上式大括号内是原三重对易括号的结果. 同理容易证明对 $m_{1}$ 求和记号中上限可换为 $k+1$, 因而上式求和号可化为 


$$
\sum_{k} \sum_{m_{1}=0}^{k+1} l_{1}^{m_{1}} \sum_{m_{2}=0}^{k+1-m_{1}} l_{2}^{m_{2}} \frac{l_{3}^{\left(k-m_{1}-m_{2}\right)+1}-l_{1}^{\left.k-m_{1}-m_{2}\right)+1}}{l_{3}-l_{4}} \delta_{k}^{(k)} A_{\xi},
$$

但由于当 $m_{1}-k+1$ 时, 对 $m_{2}$ 求和号内贡献为零, 故对 $m_{1}$ 的求和号只须求到 $k$. 然后, 我们 可以看到对 $m_{2}$ 的求和上限只须求到 $k-m_{1}$ (因为 $l_{3}^{0}-l_{4}^{0}-0$ ), 这样便得到形如

的展开式, 最后有

$$
\sum_{k} \sum_{m_{1}=0}^{k} l_{1}^{m_{1}} \sum_{m_{2}=0}^{k-m_{2}} l_{2}^{m_{2}} \sum_{m_{3}=0}^{k-m_{1}-m_{2}} l_{3}^{m_{3}} l_{1}^{k-m_{1}-m_{2}-m_{3} \delta_{\xi}^{(k)} A_{\xi} .}
$$

$$
\left[\left[\left[\delta_{a_{1}}^{\left(m_{1}\right)}, \delta_{a_{2}}^{\left(m_{2}\right)}\right], \delta_{a_{4}}^{\left(m_{3}\right)}\right], \delta_{\theta_{1}}^{\left(m_{1}\right)}\right] A_{\xi}-(-1) C_{a_{1} \xi_{2}}^{b_{1}} C_{b_{1} e_{9}}^{b_{2}} C_{b_{2} \alpha_{0}}^{\delta} \delta_{\xi}^{\left(m_{1}+m_{2}+m_{3}+m_{4}\right)} A_{\xi} .
$$

以下我们利用恒等式

$$
\begin{aligned}
& \frac{1}{\left(l_{2}-l_{1}\right)\left(l_{3}-l_{1}\right) \cdots\left(l_{n}-l_{1}\right)}+\frac{1}{\left(l_{1}-l_{2}\right)\left(l_{3}-l_{2}\right) \cdots\left(l_{n}-l_{2}\right)} \\
& +\cdots \cdots+\frac{1}{\left(l_{1}-l_{n}\right)\left(l_{2}-l_{n}\right) \cdots\left(l_{n-1}-l_{n}\right)}-0 .
\end{aligned}
$$

将上述结论推广到任意 $n$ 阶, 亦即用数学归纳法证明 $n$ 阶对易子的关系式.

我们将证明对 $n$ 阶对易子成立

$$
\begin{aligned}
& {\left[\cdots\left[\cdots\left[\delta_{\alpha_{1}}\left(l_{1}\right), \delta_{\alpha_{2}}\left(l_{2}\right)\right], \cdots\right], \delta_{a_{n}}\left(l_{n}\right)\right] A_{\xi}=\alpha_{1}^{a_{1} \alpha_{2}^{a_{2}} \cdots \alpha_{n}^{\alpha_{n}} \cdot}}
\end{aligned}
$$

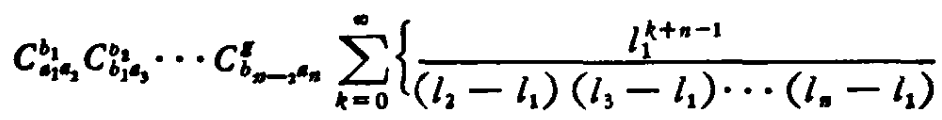

$$
\begin{aligned}
& +\frac{l_{2}^{k+n-1}}{\left(l_{1}-l_{2}\right)\left(l_{3}-l_{2}\right) \cdots\left(l_{n}-l_{2}\right)}+\cdots \\
& \left.+\frac{l_{n}^{k+n-1}}{\left(l_{1}-l_{n}\right)\left(l_{2}-l_{n}\right) \cdots\left(l_{n-1}-l_{n}\right)}\right\} \delta_{g}^{(k)} A_{\xi} \text {. }
\end{aligned}
$$

显然, 当 $n$ 取 $2,3,4$ 时正是以上得到的结果. 以下我们假定(27)式当 $n-1$ 时为正确, 然后证明对任意 $n$ 为正确. 利用恒等式(26)、(27)式中的求和号内的项可化为

利用二项式展开,注意在

$$
\begin{aligned}
I_{n}- & \sum_{k=0}^{\infty}\left\{\frac{l_{2}^{k+n-1}-l_{1}^{k+n-1}}{\left(l_{1}-l_{2}\right)\left(l_{3}-l_{2}\right) \cdots\left(l_{n}-l_{2}\right)}+\cdots\right. \\
& \left.+\frac{l_{n}^{k+n-1}-l_{n}^{k+n-1}}{\left(l_{1}-l_{n}\right)\left(l_{2}-l_{n}\right) \cdots\left(l_{n-1}-l_{n}\right)}\right\},
\end{aligned}
$$

$$
\begin{aligned}
I_{n}= & -\sum_{k=0}^{k+n} \sum_{m_{1}=0}^{k-2} l_{1}^{m_{1}}\left\{\frac{l_{2}^{k+n-2-m_{1}}}{\left(l_{3}-l_{2}\right)\left(l_{4}-l_{2}\right) \cdots\left(l_{n}-l_{2}\right)}+\cdots\right. \\
& \left.+\frac{l_{n}^{k+n-2-m_{1}}}{\left(l_{2}-l_{n}\right)\left(l_{3}-l_{n}\right) \cdots\left(l_{n-1}-l_{n}\right)}\right\} \delta_{g}^{(k)} A_{\xi}
\end{aligned}
$$

的大括号内正是 $(n-1)$ 阶的情况. 由于 $(n-1)$ 阶形式的恒等式(26)仍然成立, 所以 对 $m_{1}$ 求和的上限是 $m_{1}-k+n-3$, 而类似于 $n-4$ 时的讨论可以重复进行, 即在用二项式 展开时, 不断进行求和, 考虑到(26)式对任意 $n$ 成立, 以及 $\sum_{m_{i}=0}^{0}-0$, 重复 $n-1$ 次, 同时注 意每操作一次出现 $(-1)$ 因子,于是得到

$$
\left.\left[\left[\cdots\left[\delta_{a_{1}}\left(l_{1}\right), \delta_{a_{2}}\left(l_{2}\right)\right],\right] \cdots\right], \delta_{a_{n}}\left(l_{n}\right)\right] A_{\xi}
$$




$$
\begin{aligned}
& -(-1)^{n-1} \alpha_{1}^{a_{1}} \cdots \alpha_{n}^{a_{n}} C_{a_{1} a_{2}}^{b_{1}} C_{b_{1} a_{3}}^{t_{2}} \cdots C_{b_{n-2} a_{n}}^{g} \sum_{k=0}^{\infty} \sum_{m_{1}=0}^{k} l_{1}^{m_{1}} \sum_{m_{2}=0}^{k-m_{1}} l_{2}^{m_{2}} \sum_{m_{3}=0}^{k-m_{1}-m_{2}} l_{3}^{m_{3}} \cdots \\
& \sum_{m_{n-1}=0}^{k-m_{1}-\cdots-m_{n-2}} l_{n-1}^{m_{n-1}-1} l_{n}^{\left(k-m_{1}-\cdots-m_{n-2}-m_{n-1}\right)^{(k)}} \delta_{k} .
\end{aligned}
$$

于是得到

$$
\begin{aligned}
& {\left[\left[\cdots\left[\delta_{a_{1}}^{\left(m_{1}\right)}, \delta_{a_{2}}^{\left(m_{2}\right)}\right], \cdots\right], \delta_{a_{n}}^{(m n)}\right] A_{\xi}-(-1)^{n-1} C_{a_{1} a_{2}}^{b_{1}} C_{b_{1} a_{3}}^{b_{2}} \cdots C_{b_{n-2, n}}} \\
& \text { - } \delta_{\varepsilon}^{\left(m_{1}+m_{2}+\cdots+m_{n}\right)} A_{\xi} \text {. }
\end{aligned}
$$

它正是 (27) 式当 $n$ 取 $n-1$ 时的形式中的 $n$ 阶表达式. 于是用数学归纳法证明了(27) 形式的正确性.

以上结论完全可以推广到 SDYM 场的 $\mathrm{SL}\left(N_{1} C\right)$ 的情况. 此时 Lax-pair 为 ${ }^{[7]}$

$$
\partial_{y} U=-\lambda\left(\partial_{x}+B_{x}\right) U, \partial_{z} U=\lambda\left(\partial_{y}+B_{y}\right) U,
$$

其中

$$
\begin{aligned}
& B_{y}=J^{-1} J, y, B_{9}=J J_{.}^{-1} \bar{y}, \\
& B_{z}=J^{-1} J, z, B \bar{z}=J J^{-1} \bar{z},
\end{aligned}
$$

$y, \bar{y}, z$ 与 $\bar{z}$ 为文献 [7]中引入的变量.由文献 [7、9]知

$$
\delta B_{y}=-\frac{1}{\lambda} \partial_{z} \Lambda, \quad \delta B_{z}=\frac{1}{\lambda} \partial_{y} \Lambda \text {. }
$$

再利用文献 [7、9] 中证明的式子

$$
\delta^{\prime} U U^{-1}-\frac{\lambda}{\lambda-\lambda^{\prime}}\left(\Lambda^{\prime}-\Lambda\right), \delta U^{\prime} U^{\prime-1}-\frac{\lambda^{\prime}}{\lambda-\lambda^{\prime}}\left(\Lambda^{\prime}-\Lambda\right) .
$$

(为简单起见, 上面诸式末具体指明的无穷小参数, 可以任意选择), 由上易见现在 $B_{y}$, 代替了 主手征场 $A_{\xi}$ 的地位, 而且由于在主手征场中当 $l \rightarrow-l, \xi \rightarrow \eta$ 时整个讨论不变所以上述所 有计算都可以移到 $\mathrm{SL}(N, C)$ 情况的 SDYM 中去.

注意到这些对应，经过较䇣的计算可以得到对 $B_{y}$ 或 $B_{z}$ 的象 (30)式一样的表达式. 由于 篇幅所限, 我们不再重复这些计算的过程.

当然,如何将以上结果推广到 $G \otimes C\left(t, t^{-1}\right)$ 代数，像在文献 [10-12] 所讨论的形式的多 重对易关系,则是很繁的一个问题.

致谢: 作者感谢乔玲丽 (L.-L. Chau) 的帮助与讨论.

[1]乔玲丽,广州基本柆子物理会议文集, 1980.

[2] Pohlmeyer, K., Commun. Math. Phys., 72(1980), 37.

[ 3 ] Dolan, L. \& Roos, A., Phys. Rev., D22(1980), 2018.

[ 4 ] Kae, V. G., Math. USSR-Izv., 2(1968), 1311; Moody, R. V., J. Algebra, 10(1968), 211.

[5] Hou, B. Y., Ge, M. L. \& Wu, Y. S., Phys. Rev., D24(1981), 2238.

[6] Ge, M. L. \& Wu, Y. S., Phys. Lett., 108B(1982), 411.

[7] Chau, I. -L., Ge, M. L. \& Wu, Y. S., Phys. Rev., D25(1982), 1086.

[ 8 ] Devehand, C. \& Fairlie, D. B., Nucl. Phys., B194 (1982), 232.

[0] 悬林、吴铍时、科学通报, 27 (1982), 24: 1486.

[10] Wu, Y. S., Preprint, Institute for Advanced Study, 1982.

[11] Chau, L.-L. \& Wu, Y. S., BNL Preprint, 1982.

[12] Chau, L.-L., Ge, M. L., Sinha, A. \& Wu Y. S., ibid., 1982.

第 11 期

科学 通报 